\title{
Analisando a Capacidade de Previsão entre Diferentes Classes de Modelos: um Estudo Comparativo para os Índices de Produção Industrial no Brasil
}

\author{
Felipe Leite Coelho da Silva ${ }^{1}$ \\ Departamento de Matemática/UFRRJ, Seropédica, RJ \\ Kleyton Vieira Sales da Costa ${ }^{2}$ \\ Departamento de Economia/UFRRJ, Seropédica, RJ
}

\begin{abstract}
Resumo. Os indicadores de produção industrial são variáveis de interesse para a mensuração da atividade econômica de uma determinada região ou país e, assim, podem ser instrumentos de apoio à tomada de decisão para legisladores e agentes econômicos em geral. Desse modo, este trabalho propõe a utilização de três classes de modelos de previsão de séries temporais: modelos clássicos Holt-Winters e SARIMA; de espaço de estado - modelo linear dinâmico e TBATS; e redes neurais artificiais (NNAR - neural network autoregression e MLP - multilayer perceptron). Os modelos foram aplicados aos indicadores de produção industrial do Brasil para a indústria extrativa e de transformação. Através das métricas de precisão RMSE e MAPE, o modelo MLP foi o que obteve o melhor desempenho para ambos os conjuntos de dados em análise.
\end{abstract}

Palavras-chave. Séries Temporais, Previsão, Redes Neurais, Produção Industrial

\section{Introdução}

Os índices de produção industrial atuam como instrumentos de suporte à tomada de decisão e termômetros da atividade econômica de uma determinada região ou país. Desde a década de 1970, os indicadores para a produção industrial brasileira são estabelecidos a partir da Pesquisa Industrial Mensal-Produção Física (PIM-PF) [7], tendo como finalidade mensurar as variações do valor adicional criado pelo processo de produção em um dado período. Por definição, o valor adicional criado pelo processo de produção é caracterizado como valor adicionado, bruto ou líquido. Valor adicionado bruto é o valor bruto da produção menos o consumo intermediário e o valor adicionado líquido é o valor bruto da produção menos o consumo intermediário e o consumo de capital fixo. Dessa maneira, até o momento atual, a PIM-PF monitora a produção industrial através das atividades econômicas que estão dentro do escopo de análise da Pesquisa Industrial Anual (PIA) - Indústria Extrativa e de Transformação.

As atividades da indústria extrativa que são consideradas pela PIA seguem o estabelecido pela Classificação Nacional de Atividades Econômicas (CNAE), ou seja, as atividades produtivas de extração dos minerais em seu estado natural. Dentro dessa classificação são considerados todos aqueles minerais que estejam em seu estado sólido, liquido e gasoso. Com isso, estão dentro da esfera da pesquisa os processos produtivos que tenham por finalidade a extração de carvão, minerais de forma geral, petróleo cru e gás natural. Para a indústria de transformação, considera-se todo

\footnotetext{
${ }^{1}$ felipeleiterural@gmail.com

2kleyton.vsc@gmail.com
} 
tipo de atividade que gere novos produtos a partir da modificação química, física e biológica dos insumos primários produzidos através de outras atividades industriais. Uma limitação para o enquadramento de uma indústria como sendo de transformação está na dificuldade em se estabelecer o que seria um produto novo. Para ultrapassar esta barreira, existem definições convencionadas, explicitadas em subsetores a partir da CNAE como, por exemplo, a fabricação de produtos alimentícios, fabricação de bebidas, confecção de artigo de vestuário, entre outros.

As contribuições da literatura que abordam a previsão de indicadores de produção industrial aplicam diferentes estratégias de modelagem. O estudo desenvolvido em [1] leva em consideração uma abordagem para o método de previsão de fatores. Esse trabalho investiga a capacidade de previsão destes métodos para uma série de variáveis econômicas, incluindo a taxa de crescimento da produção industrial. As conclusões apontam para resultados de previsão satisfatórios quando são utilizados preditores direcionados, sendo uma estratégia útil na redução da raiz do erro quadrático médio em algumas das variáveis macroeconômicas consideradas; [2] utiliza modelos fatoriais de alta dimensão na previsão de variáveis macroeconômicas, entre elas o índice de produção industrial do Brasil. Os resultados indicam que o índice de produção industrial obteve melhores resultados de previsão a partir de métodos fatoriais sem a introdução de aprendizado estatístico; [4] tem como objetivo prever o crescimento da produção industrial e da inflação na Turquia. Foram utilizadas as abordagem de modelos fatoriais e combinação de previsões. Neste estudo, os resultados indicam a superioridade preditiva dos modelos de fatores em relação à combinação de modelos, para as variáveis em análise; [8] investiga a capacidade preditiva dos modelos de redes neurais artificiais para o índice de produção industrial da Índia. Os resultados apontam que um modelo não-linear de redes neurais autorregressivo com entradas exógenas (NARX) obteve o resultado de previsão mais satisfatório; e [9] investiga a utilização de variáveis desagregadas para a previsão da produção industrial brasileira, encontrando resultados que apontam para uma melhor capacidade preditiva dos modelos quando se leva em consideração dados desagregados.

A finalidade deste trabalho é propor um estudo comparativo entre três classes de modelos de previsão de séries temporais (clássicos, de espaço de estado e de redes neurais artificiais) aplicados aos índices de produção industrial do Brasil. Para atingir este objetivo, foram considerados o método de Holt-Winters e o modelo SARIMA, como parte da abordagem clássica; para a abordagem de espaço de estado, o modelo linear dinâmico e TBATS; e os modelos de redes neurais artificiais NNAR (neural network autoregression) e MLP (multilayer perceptron). Destaca-se que a utilização destas classes de modelos dentro de um mesmo estudo comparativo não foi encontrada na literatura, ainda que, como observado, existam estudos que apliquem modelos de previsão aos indicadores de produção industrial do Brasil. Portanto, o desenvolvimento deste trabalho pode contribuir para a literatura disponível nesta área.

Além desta primeira seção de introdução, o trabalho está organizado da seguinte maneira: a seção 2 apresenta a metodologia utilizada para a investigação empírica, a seção 3 traz os resultados e discussões e, por fim, a seção 4 apresenta as considerações finais e propostas para pesquisas futuras.

\section{Metodologia}

A abordagem empírica leva em consideração os dados do índice de Produção Industrial no Brasil (Indústria Extrativa e Indústria de Transformação), extraídos a partir da base de dados do Sistema IBGE de Recuperação Automática (SIDRA). Os dados foram separados em conjunto de treino (dezembro de 2003 a dezembro de 2017) para ajustar os modelos e conjunto de teste (janeiro de 2018 a dezembro de 2019) para avaliar os modelos quanto à qualidade na previsão. O período de tempo observado abrange a crise financeira mundial iniciada em 2008 e com reflexos na economia real a partir de 2009 .

A seleção do modelo com o melhor desempenho foi estabelecida através das métricas de precisão MAPE (erro médio absoluto percentual) e RMSE (raiz do erro quadrático médio). 
Todas as análises estatísticas foram desenvolvidas através das linguagem de programação $\mathrm{R}$ 4.0.5 (The R Foundation for Statistical Computing, Vienna, Austria; http://www.r-project.org).

\subsection{Descrição dos Modelos}

Esta seção descreve os modelos que foram propostos para investigação: método de Holt-Winters, modelo SARIMA, modelo linear dinâmico, TBATS, redes neurais autorregressivas e redes neurais com perceptron multicamadas.

\subsubsection{Método de Holt-Winters}

De acordo com [6], o método aditivo de Holt-Winters tem a sua componente de sazonalidade definida em termos absolutos de escala para a série observada. A equação de nível deste método possui um ajuste sazonal da série pela subtração da componente de sazonalidade e dentro de cada período a componente sazonal tem uma soma de aproximadamente zero. Para o método multiplicativo, a componente de sazonalidade é definida em termos percentuais e a série tem o seu ajuste sazonal através da sua divisão pela componente sazonal. Assim, cada período tem uma soma de aproximadamente m.

Os métodos aditivo e multiplicativo (Equação 1) de Holt-Winters são definidos a partir de três equações de suavização e uma equação de previsão, definidas como

$$
\begin{aligned}
& \hat{y}_{t+h \mid t}^{(a)}=\ell_{t}+h b_{t}+s_{t+h-m(k+1)} \\
& \hat{y}_{t+h \mid t}^{(m)}=\left(\ell_{t}+h b_{t}\right) s_{t+h-m(k+1)} \\
& \ell_{t}^{(a)}=\alpha\left(y_{t}-s_{t-m}\right)+(1-\alpha)\left(\ell_{t-1}+b_{t-1}\right) \\
& \ell_{t}^{(m)}=\alpha \frac{y_{t}}{s_{t-m}}+(1-\alpha)\left(\ell_{t-1}+b_{t-1}\right) \\
& b_{t}=\beta\left(\ell_{t}-\ell_{t-1}\right)+(1-\beta) b_{t-1} \\
& s_{t}^{(a)}=\gamma\left(y_{t}-\ell_{t-1}-b_{t-1}\right)+(1-\gamma) s_{t-m} \\
& s_{t}^{(m)}=\gamma \frac{y_{t}}{\left(\ell_{t-1}+b_{t-1}\right)}+(1-\gamma) s_{t-m}
\end{aligned}
$$

em que $l_{t}^{(a, m)}, b_{t}, s_{t}^{(a, m)}$ são as equações de suavização, respectivamente, para as componentes de nível, tendência e sazonalidade. E a equação $\hat{y}_{t+h \mid t}$ é a equação de previsão do modelo, sendo desenvolvida de acordo com o método empregado - aditivo (a) ou multiplicativo (m).

\subsubsection{SARIMA}

Modelos de Box \& Jenkins determinam o processo estocástico adequado para representar uma determinada série temporal a partir da passagem de um ruído branco por um filtro linear. O modelo utilizado foi o SARIMA (sazonal autorregressivo integrado de médias móveis), buscando incorporar a componente de sazonalidade que está presente nos dados em análise. O modelo SARIMA de ordem $(p, d, q) \times(P, D, Q)_{s}$ é definido por,

$$
\phi(B) \Phi\left(B^{s}\right) \nabla^{d} \nabla_{s}^{D} Y_{t}=\theta(B) \Theta\left(B^{s}\right) \alpha_{t}
$$

em que $\phi(B)$ é o operador autorregressivo simples, $\Phi\left(B^{s}\right)$ é o operador autorregressivo sazonal, $\nabla^{d}$ é o operador da diferença simples, $\nabla_{s}^{D}$ é o operador da diferença sazonal, $\theta(B)$ é o operador de médias móveis simples, $\theta\left(B^{s}\right)$ é o operador de médias móveis sazonal e $\alpha_{t}$ é um erro aleatório.

\subsubsection{Modelo Linear Dinâmico}

Para cada período de tempo t, o modelo linear dinâmico univariado em sua forma generalizada é definido através das equações $Y_{t}$ e $\theta_{t}$ (Equação 3) [10]. 
Equação observacional: $Y_{t}=F_{t} \theta_{t}+v_{t}, \quad v_{t} \sim N_{m}\left(0, V_{t}\right)$

Equação de sistema: $\theta_{t}=G_{t} \theta_{t-1}+w_{t}, \quad w_{t} \sim N_{p}\left(0, W_{t}\right)$.

\subsubsection{TBATS}

O modelo TBATS faz uso de uma combinação de termos de Fourier com um modelo de espaço de estado de suavização exponencial e transformação Box-Cox, sendo útil por se ajustar a mudança na sazonalidade ao longo do tempo. Proposto em [3], o modelo pode ser escrito em sua forma reduzida a partir da equação

$$
\varphi_{p}(L) \eta(L) y_{t}^{(\omega)}=\theta_{q}(L) \delta(L) \varepsilon_{f}
$$

em que $L$ é o operador de defasagem, $\eta(L)=\operatorname{det}\left(\mathbf{I}-\mathbf{F}^{*} L\right), \delta(L)=\mathbf{w}^{*} \operatorname{adj}\left(\mathbf{I}-\mathbf{F}^{*} L\right) \mathbf{g}^{*} L+$ $\operatorname{det}\left(\mathbf{I}-\mathbf{F}^{*} L\right), \varphi_{p}(L)$ e $\theta_{q}(L)$ são polinômios de grau $p$ e $q, \mathbf{w}^{*}=(1, \phi, \mathbf{a}), \mathbf{g}^{*}=(\alpha, \beta, \gamma)^{\prime}$, sendo a matriz $\mathbf{F}^{*}$ definida por

$$
\mathbf{F}^{*}=\left[\begin{array}{lll}
1 & \phi & 0 \\
0 & \phi & 0 \\
0^{\prime} & 0^{\prime} & \mathbf{A}
\end{array}\right]
$$

\subsubsection{Redes Neurais Artificiais}

Um modelo de redes neurais artificiais busca modelar a relação entre um conjunto de sinais de entrada e um sinal de saída. Neste estudo foram considerados os modelos de redes neurais autorregressivas (NNAR) e multilayer perceptron (MLP). O modelo $N N A R(p, P, k)_{m}$ considerado leva em consideração uma rede feed-forward com uma camada oculta, $p$ entradas, $k$ nós na camada oculta, $P$ defasagens sazonais e $m$ períodos. O modelo MLP é um dos modelos de redes neurais artificiais mais utilizados. A sua arquitetura está dividida entre a camada de entrada, a camada oculta e a camada de saída [5], como observado na Figura 1.

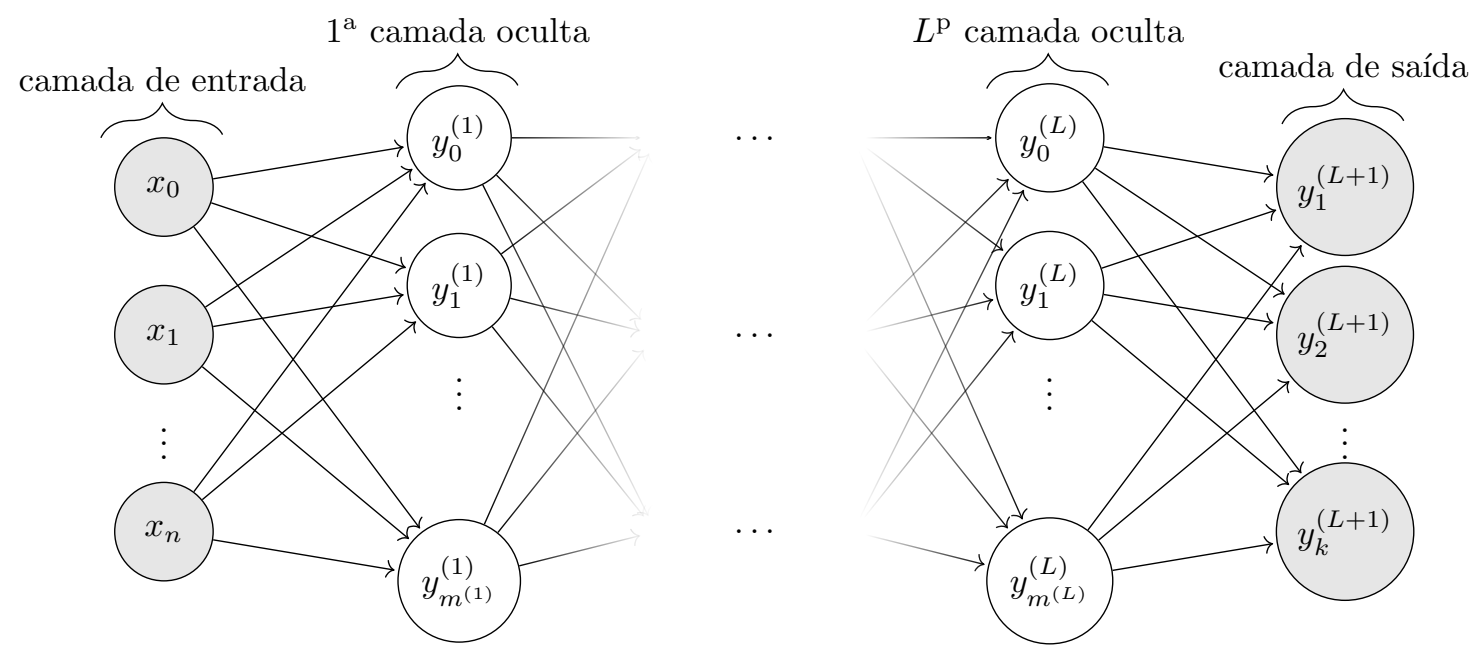

Figura 1: Rede neural MLP de $(L+1)$-camadas com $n$ unidades de entradas e $k$ unidades de saída. A $L^{\mathrm{p}}$ camada oculta contém $m^{(L)}$ unidades ocultas.

\section{Resultados}

As Figuras 2 e 3 apresentam o ajuste dos modelos aos dados mensais do índice de produção da indústria de transformação e extrativa entre 2003 e 2017, assim como a previsão mensal para 
o horizonte de dois anos à frente. Pode-se observar nos gráficos que o ajuste dos modelos em relação aos dados foi satisfatório, mostrando a capacidade de adaptação dos modelos em relação a estrutura dos dados, tanto para os períodos de crise e expansão da atividade econômica como na assimilação das componentes de tendência e sazonalidade.

(a) Ajuste

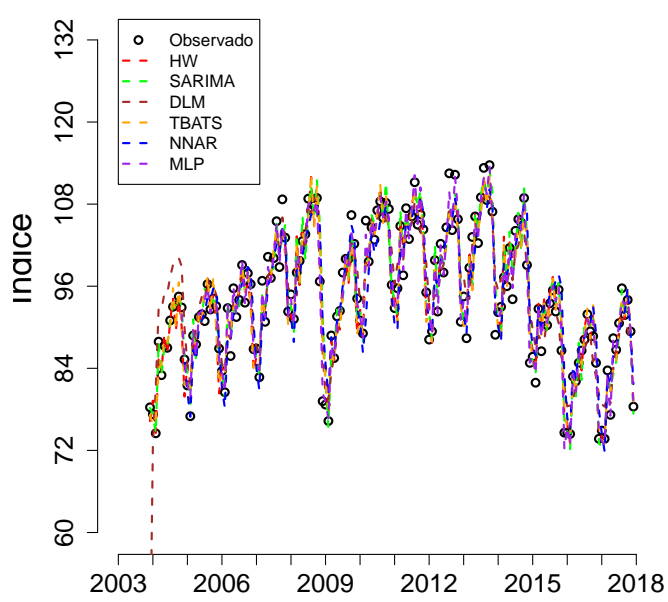

(b) Previsão

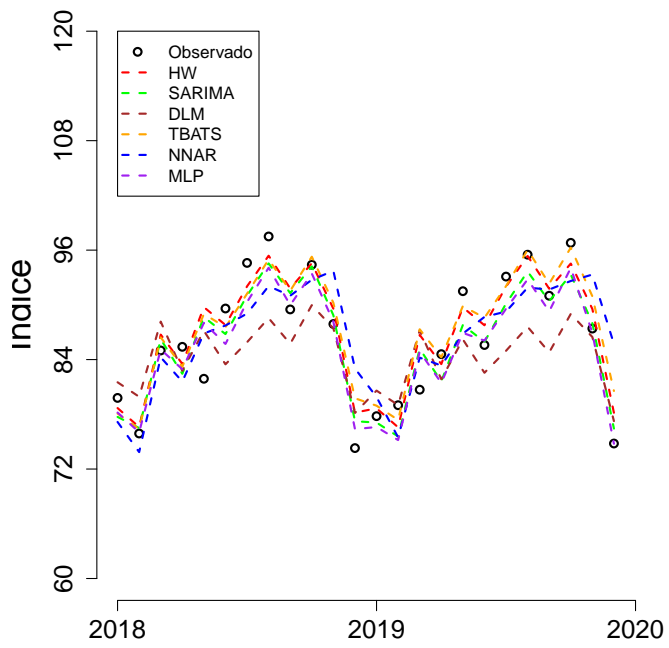

Figura 2: Resultados de ajuste (a) e previsão (b) para o indicador de produção da indústria de transformação no Brasil.

(a) Ajuste

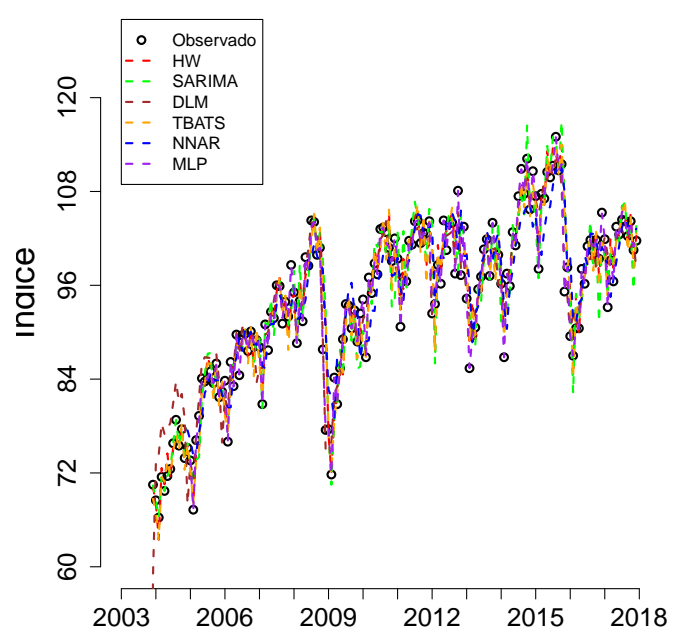

(b) Previsão

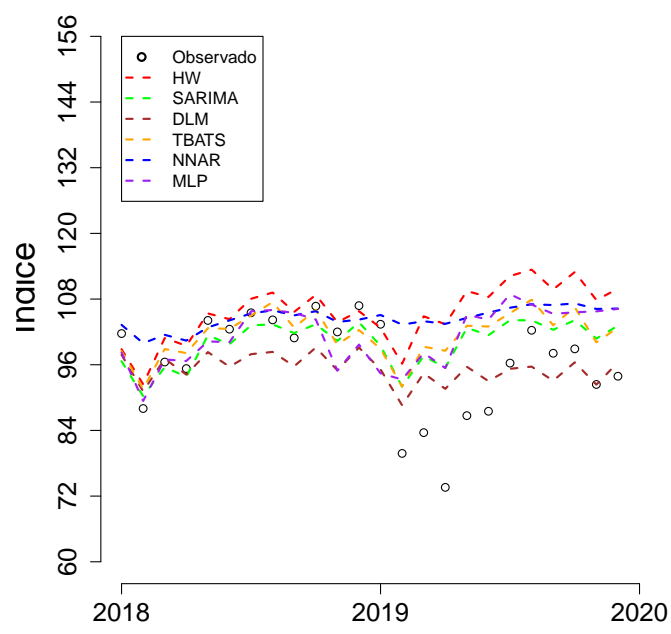

Figura 3: Resultados de ajuste (a) e previsão (b) para o indicador de produção da indústria extrativa no Brasil. 
A Tabela 1 mostra a comparação entre os modelos ajustados usando os critérios de avaliação de acurácia (RMSE, MAPE). De acordo com os critérios de acurácia, todos os modelos apresentaram um resultado expressivo no sentido de atestar a qualidade de ajuste dos modelos aos dados. Nos resultados, o MAPE de ajuste dos modelos ao conjunto de treino foi inferior a $2 \%$ (Tabela 1). Contudo, observa-se que o melhor desempenho foi obtido através do modelo MLP, tanto para o indicador de produção industrial da indústria de transformação como para a indústria extrativa.

Tabela 1: Métricas de ajuste.

\begin{tabular}{lcccc}
\hline Modelo & RMSE* & MAPE* & RMSE** $^{*}$ & MAPE** \\
\hline Holt-Winters & 0.2882 & 1.2888 & 0.1313 & 0.8753 \\
SARIMA & 0.2770 & 1.2037 & 0.1658 & 1.0825 \\
Linear Dinâmico & 0.5409 & 1.9712 & 0.1581 & 1.1438 \\
TBATS & 0.2821 & 1.3063 & 0.1339 & 0.9447 \\
NNAR & 0.3364 & 1.4483 & 0.1986 & 1.4260 \\
MLP & $\mathbf{0 . 1 3 4 3}$ & $\mathbf{0 . 5 5 3 6}$ & $\mathbf{0 . 0 0 8 5}$ & $\mathbf{0 . 0 3 2 4}$ \\
\hline
\end{tabular}

*Indústria de Transformação

**Indústria Extrativa

Os resultados dos modelos para o horizonte de previsão, vinte e quatro meses à frente, são apresentados na Tabela 2. Pelos critérios de acurácia, os modelos apresentaram uma boa capacidade preditiva para indústria de transformação. E, para a indústria extrativa, o modelo linear dinâmico e MLP apresentaram os melhores resultados para previsão. Assim como no caso do conjunto de treino, o modelo MLP obteve os melhores resultados para o conjunto de teste, superando todos os outros modelos propostos ao analisarmos o erro absoluto percentual médio (Tabela 2).

Tabela 2: Métricas de previsão.

\begin{tabular}{lcccc}
\hline Modelo & RMSE* & MAPE* & RMSE** $^{* *}$ & MAPE** $^{* *}$ \\
\hline Holt-Winters & 0.2994 & 1.4345 & 0.5244 & 3.7589 \\
SARIMA & 0.3001 & 1.5133 & 0.4021 & 2.8253 \\
Linear Dinâmico & 0.5479 & 2.8457 & $\mathbf{0 . 3 3 9 3}$ & 2.6592 \\
TBATS & 0.3390 & 1.6271 & 0.4387 & 3.0830 \\
NNAR & 0.5567 & 2.5933 & 0.5597 & 3.9197 \\
MLP & $\mathbf{0 . 2 7 7 0}$ & $\mathbf{1 . 3 0 7 6}$ & 0.3668 & $\mathbf{2 . 3 8 8 4}$ \\
\hline
\end{tabular}

*Indústria de Transformação

**Indústria Extrativa

\section{Conclusões}

A indústria é um dos setores da economia que contribui de maneira expressiva para a geração de emprego e renda. Além disso, o comportamento dos indicadores de produção industrial é um elemento eficiente como auxiliar na tomada de decisão de legisladores, investidores domésticos ou estrangeiros, entre outros. Com isso, o objetivo deste trabalho foi propor um estudo comparativo entre as classes de modelos clássicos, de estado de espaço e de redes neurais, aplicados aos indicadores de produção industrial do Brasil, na busca por um modelo que obtivesse resultados satisfatórios de ajuste e previsão em relação aos dados observados. Foi considerado a capacidade 
preditiva de seis modelos: método de Holt-Winters, SARIMA, Linear Dinâmico, TBATS, NNAR e MLP.

Os resultados do estudo indicam que: (i) os modelos considerados possuem uma capacidade de ajuste aos dados satisfatória; (ii) os modelos conseguiram capturar a estrutura complexa dos dados que envolvem as crises (picos nas séries) nos anos de 2009 e 2016; e (iii) o modelo MLP apresentou a melhor capacidade preditiva para o grupo de modelos propostos. Além disso, os resultados encontrados são úteis como instrumentos de apoio à tomada de decisão por parte dos agentes econômicos e legisladores, contribuindo para a construção de políticas públicas baseadas em evidências e melhorando a qualidade dos investimentos no Brasil.

Um estudo interessante para pesquisas futuras seria investigar e comparar os modelos Recurrent Neural Networks (RNN), Long short-term memory (LSTM) e Convolutional Neural Networks $(\mathrm{CNN})$.

\section{Referências}

[1] Bai, J. Forecasting economic time series using targeted predictors, Journal of Econometrics, 2008. DOI: $10.1016 / \mathrm{j} . j e c o n o m .2008 .08 .010$.

[2] Barbosa, Rafael B. and Ferreira, Roberto Tatiwa and Silva, Thibério Mota da.. Previsão de Variáveis Macroeconômicas Brasileiras usando Modelos de Séries Temporais de Alta Dimensão., Estudos Econômicos.,vol.50, n.21, 2020. DOI: 10.1590/0101-41615013rrt.

[3] De Livera, A. M. and Hyndman, R. J. and Snyder, R. D.. Forecasting Time Series With Complex Seasonal Patterns Using Exponential Smoothing., Journal of the American Statistical Association., 106:496, 2011. DOI: 10.1198/jasa.2011.tm09771.

[4] Gunay, M.. Forecasting industrial production and inflation in Turkey with factor models, Central Bank Review, 2018. DOI: 10.1016/j.cbrev.2018.11.003.

[5] Haykin, Simon. Neural networks and learning machines, 3a. edição. Pearson, 2008.

[6] Hyndman, R. J., and Athanasopoulos, G. Forecasting: principles and practice, 3a. edição. OTexts, Melbourne, 2021.

[7] IBGE, Coordenação de Indústria Indicadores conjunturais da indústria : produção, 2a. edição. IBGE, Rio de Janeiro, 2015.

[8] Potdar, Kedar and Kinnerkar, Rishab. A non-linear autoregressive neural network model for forecasting Indian index of industrial production, 2017 IEEE Region 10 Symposium (TENSYMP) pages 1-5, 2017. DOI: 10.1109/TENCONSpring.2017.8069973.

[9] Prince, D. and Marçal, E. F..Selection of lags and univariate models to forecast industrial production in an Emerging country: is disagreggation useful?, Encontro Nacional de Economia, 2018.

[10] West, M. and Harrison, J. Bayesian forecasting and dynamic models, 3a. edição. Springer Science, 2006. 